\title{
Recent Advances in Fluorescence Imaging of Pulmonary Fibrosis in Animal Models
}

\author{
Zongwei Liu ${ }^{1}$, Xiaofang Tang ${ }^{2}$, Zongling Zhu ${ }^{3}$, Xunxun $\mathrm{Ma}^{1}$, Wenjuan $\mathrm{Zhou}^{4 *}$ and \\ Weijiang Guan ${ }^{5 *}$
}

${ }^{1}$ Department of Respiratory Medicine, Lianyungang Hospital of Traditional Chinese Medicine (TCM), Affiliated Hospital of Nanjing University of Chinese Medicine, Lianyungang, China, ${ }^{2}$ Green Catalysis Center, College of Chemistry, Zhengzhou University, Zhengzhou, China, ${ }^{3}$ Department of Respiratory Medicine, Pukou District Hospital of Chinese Medicine, Pukou Branch of Nanjing Hospital of Chinese Medicine, Affiliated to Nanjing University of Chinese Medicine, Nanjing, China, ${ }^{4}$ Department of Chemistry, Capital Normal University, Beijing, China, ${ }^{5}$ State Key Laboratory of Chemical Resource Engineering, College of Chemistry, Beijing University of Chemical Technology, Beijing, China

\section{OPEN ACCESS}

Edited by:

Ruilin Liu,

Xuzhou Medical University, China

Reviewed by:

Guangcun Chen,

Chinese Academy of Sciences (CAS),

China

Xiaolong Sun,

X'an Jiaotong University, China

${ }^{*}$ Correspondence:

Wenjuan Zhou

zhouwenjuan@cnu.edu.cn

Weiiiang Guan

wjguan@mail.buct.edu.cn

Specialty section:

This article was submitted to

Nanobiotechnology,

a section of the journal

Frontiers in Molecular Biosciences

Received: 09 September 2021

Accepted: 18 October 2021

Published: 02 November 2021

Citation:

Liu Z, Tang X, Zhu Z, Ma X, Zhou W and Guan W (2021) Recent Advances in Fluorescence Imaging of Pulmonary

Fibrosis in Animal Models.

Front. Mol. Biosci. 8:773162.

doi: 10.3389/fmolb.2021.773162
Pulmonary fibrosis (PF) is a lung disease that may cause impaired gas exchange and respiratory failure while being difficult to treat. Rapid, sensitive, and accurate detection of lung tissue and cell changes is essential for the effective diagnosis and treatment of PF. Currently, the commonly-used high-resolution computed tomography (HRCT) imaging has been challenging to distinguish early PF from other pathological processes in the lung structure. Magnetic resonance imaging (MRI) using hyperpolarized gases is hampered by the higher cost to become a routine diagnostic tool. As a result, the development of new PF imaging technologies may be a promising solution. Here, we summarize and discuss recent advances in fluorescence imaging as a talented optical technique for the diagnosis and evaluation of PF, including collagen imaging, oxidative stress, inflammation, and PFrelated biomarkers. The design strategies of the probes for fluorescence imaging (including multimodal imaging) of PF are briefly described, which can provide new ideas for the future PF-related imaging research. It is hoped that this review will promote the translation of fluorescence imaging into a clinically usable assay in PF.

Keywords: pulmonary fibrosis, imaging, nanomaterial, biomarkers, near-infrared

\section{INTRODUCTION}

As a chronically progressive disease, pulmonary fibrosis (PF) features activation of myofibroblasts and subsequent deposition of large amounts of extracellular matrix, leading to the impaired gas exchange and the increased risk of respiratory failure (Richeldi et al., 2017; Ni et al., 2018; Hayward et al., 2021). In particular, PF is considered a risk factor for the severe evolution of coronavirus disease 2019, which is rapidly spreading and escalating into a global pandemic affecting the health of billions of people (Crisan-Dabija et al., 2020; George et al., 2020). Given that lung transplantation is the only effective treatment for PF to date (Juillerat-Jeanneret et al., 2018), the early diagnosis of PF is critical to help reduce patient morbidity and improve patient survival.

Currently, PF is primarily diagnosed by high-resolution computed tomography (HRCT) imaging (Wu et al., 2019; Mento et al., 2020). However, lung structures in early pulmonary fibrosis may not be deformed, making it difficult to distinguish fibrosis from other pathological processes (Désogère et al., 2017). HRCT also faces challenges in detecting idiopathic pulmonary fibrosis (IPF) because of its atypical appearance on HRCT images (Liu et al., 2017; Barratt et al., 2018; Ley and Ley- 
Zaporozhan, 2020). A practical alternative is to use the magnetic resonance imaging (MRI). Although the presence of an air-tissue interface usually results in a low proton density and poor magnetic susceptibility environment in the lung (Mammarappallil et al., 2019), MRI using hyperpolarized gases (e.g., helium or xenon) can reveal subtle changes in lung microstructure (Lonzetti et al., 2019). Unfortunately, the high cost constrains it from becoming a routine diagnostic tool. Therefore, the development of cost-effective and highlysensitive imaging techniques for the early diagnosis of $\mathrm{PF}$ is necessary to assess the status of the lung and to guide patients toward the appropriate clinical pathway.

Fluorescence imaging is based on the detection of electromagnetic radiation in the ultraviolet-visible-nearinfrared (UV-Vis-NIR) range emitted by fluorophores (Ximendes et al., 2021). Thanks to the advantages of high sensitivity, tunable specificity, controllable cost, acceptable biosafety, and noninvasiveness (Fatahi et al., 2020; Guan et al., 2020; Niu et al., 2020; Guo et al., 2021; He et al., 2021), fluorescence imaging has been applied to visualize vascular system (Ma et al., 2018; Zheng et al., 2019), lymphatic system (Nakajima et al., 2018), various tumor tissues (Bai et al., 2020; Bai et al., 2021), and real-time blood flow (Hong et al., 2014; Wan et al., 2018; Cai et al., 2020). In addition, fluorescence imaging shows great potential in facilitating the development of precise therapeutic strategies for diseases, including imaging-guided cell therapy, drug delivery/release monitoring, and photodynamic therapy (Li et al., 2020; Li et al., 2019a; Liu et al., 2019; Han et al., 2021; Xu et al., 2021). It is clear that fluorescence imaging can play an important role in the diagnosis, treatment, and rehabilitation of PF. Herein, we summarize the knowledge of PF-related fluorescence imaging in terms of collagen imaging, oxidative stress and inflammation imaging, PF-related biomarker imaging, and imaging-guided PF therapy. The properties and drawbacks of relevant fluorescent probes, as well as possible solutions, are briefly discussed. By reviewing these recent advances in PF-related fluorescence imaging, we hope to contribute to potential protocols and ideas for future PFrelated imaging studies.

\section{IMAGING OF COLLAGEN}

Collagen is the main structural component of the extracellular matrix in mammalian tissues. The deposition of collagen is a common feature of the PF patients (Snijder et al., 2019; Sorushanova et al., 2019; Yombo et al., 2021). Currently, collagen staining, collagen antibodies, and imaging systems (second harmonic generation and transmission electron microscopy) face the challenge of distinguishing structural changes between intact collagen and degraded collagen at the molecular level (Nielsen et al., 1998; Fligiel et al., 2003; Matteini et al., 2012; Hwang et al., 2017a). Inspired by the unique triplehelical structure of collagen, a collagen hybrid peptide (CHP) was developed to have specific binding to denatured collagen chains but negligible binding to intact collagen (Hwang et al., 2017b). By linking 5-carboxyfluorescein (green-fluorescent dye) on CHP, in situ fibrotic changes in frozen sections of lung tissues from bleomycin-treated animal models were observed by fluorescence microscopy. As shown in Figure 1A, bright spots indicated the damaged collagens bound to $\mathrm{CHP}$ in vivo. The signals in the central and subpleural areas increased over time, while the number of bright spots in the subpleural area increased more significantly, providing direct evidence for the structural abnormality of collagens in fibrotic tissues.

Compared to single-modality imaging, multi-modality imaging tends to have higher sensitivity and specificity by combining the advantages of various imaging techniques, allowing for a more comprehensive evaluation of PF-related targets (Perez et al., 2017; Ruscitti et al., 2018). For example, CT imaging could provide macroscopic anatomical information in clinical diagnosis, while fluorescence endomicroscopy (FE) using targeted probes enabled the observation of microscopic fine structures. A collagen-targeted fluorescent probe was conveniently designed by replacing the signal unit of the reported MRI probe with fluorescein isothiocyanate (FITC). The obtained Ac-Lys (Ac)-Trp-His- $\left[{ }^{\star}\right.$ Cys-Thr-Thr-K(FITC)Phe-Pro-His-His-Tyr-Cys]-Leu-Tyr-Bip-Amide showed satisfactory binding affinity to collagen in a plate binding assay (Perez et al., 2017). Accordingly, a correlation between lung density on $\mathrm{CT}$ images and collagen fiber structures on FE images was observed at any given corresponding endomicroscopy location (Figures 1B,C). In addition, another dual-modality imaging that can evaluate the progression of bleomycininduced PF in mice was constructed by changing fluorescent probes (Ruscitti et al., 2018). Fluorescence molecular tomography (FMT) using a matrix metalloproteinase fluorescent probe could reveal quantitative information about the expression of IPF protein in vivo and in vitro, while micro-CT imaging reflected the pathological and therapeutic status of lung parenchyma. There is no doubt that PF-related multi-modality imaging has great potential and deserves more attention and effort.

\section{IMAGING OF OXIDATIVE STRESS AND INFLAMMATION}

PF patients are susceptible to high levels of oxidative stress because the lung is directly involved in respiration (Cheresh et al., 2013; He et al., 2019). Oxidative stress was positively correlated with the reactive oxygen species (ROS) levels (Schieber and Chandel, 2014; Song et al., 2021). Moreover, early inflammation in PF may also increase the ROS levels (Wang et al., 2020). Therefore, ROS-responsive fluorescent probes hold promise for the early diagnosis of PF.

Mitochondria are the main source of cellular hydrogen peroxide $\left(\mathrm{H}_{2} \mathrm{O}_{2}\right)$ (Wei et al., 2021). Mitochondrial-targeted fluorescent probes are more likely potential strategies for further understanding of the mechanism of the disease occurrence and progression, as well as early diagnosis and treatment of the disease. A simple and effective method is to introduce mitochondrial-targeted groups (triphenylphosphonium) into the fluorescent scaffold (Song et al., 2021). Selective detection of mitochondrial $\mathrm{H}_{2} \mathrm{O}_{2}$ was 
A
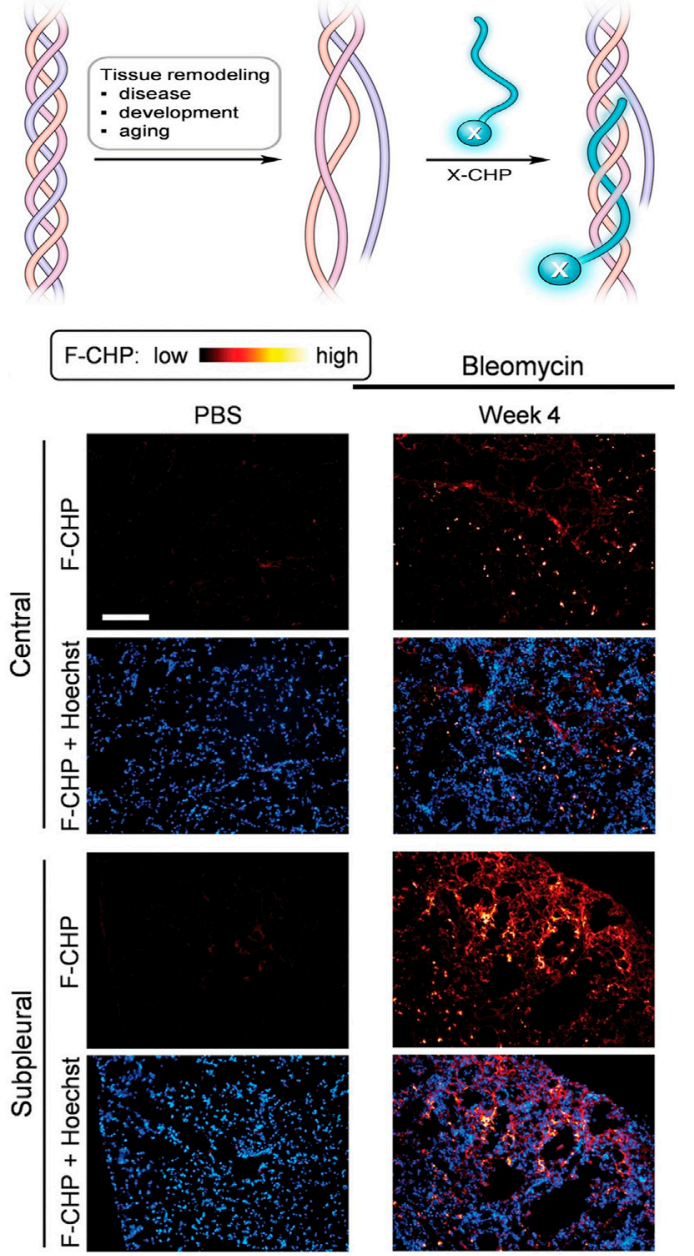

Week 4
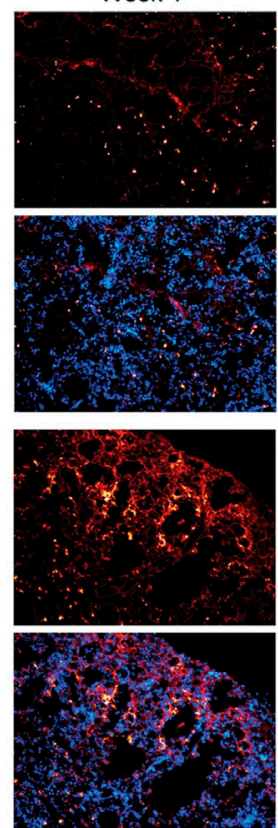

B

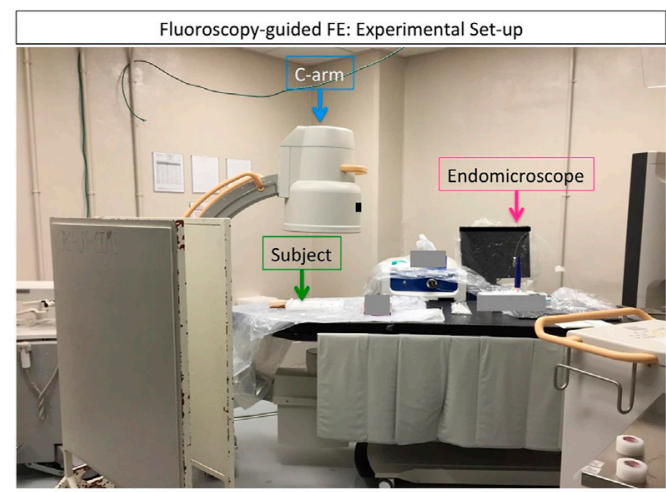

C
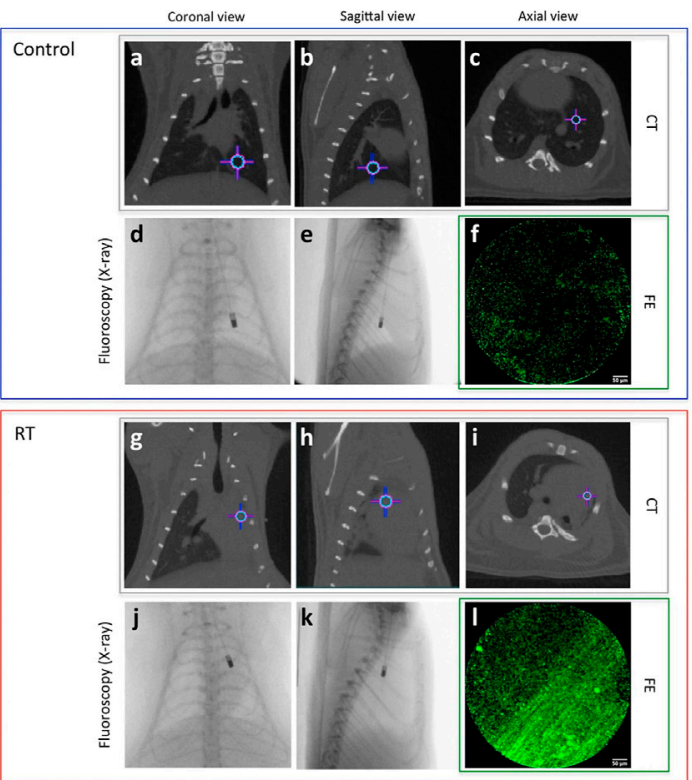

FIGURE 1 | (A) Schematic of a CHP strand (labeled with X) hybridizing to denatured collagen chains and forming a collagen triple helix and representative fluorescence micrographs of the center and subpleural area of frozen lung sections, which were obtained from bleomycin mice and control mice. (B) Experimental setup. (C) Representative CT (a-c and g-i, gray box) and FE (f and I, green box) images of control (a-f, blue box) and RT (g-I, red box) at the corresponding endomicroscope position determined with the fluoroscopy images ( $d$, e and $\mathrm{j}, \mathrm{k}$, respectively). Crosshair indicates the position of the endomicroscope tip. Adapted and modified with permission from ref (Hwang et al., 2017) (Copyright 2017 American Chemical Society) and ref (Perez et al., 2017) (Copyright 2017; published by Springer Nature).

realized by the combination of the azo-BODIPY (NIR-fluorescent dye), 4-(bromomethyl) phenylboronic acid pinacol ester $\left(\mathrm{H}_{2} \mathrm{O}_{2}\right.$ response unit), and triphenylphosphonium cation (mitochondrial-targeted group) in one molecular probe (MitoBor). It showed little fluorescence because of photoinduced electron transfer (PET) between azo-BODIPY and 4(bromomethyl) phenylboronic acid pinacol ester. In the presence of $\mathrm{H}_{2} \mathrm{O}_{2}$, the NIR fluorescence $(\sim 730 \mathrm{~nm})$ of MitoBor was turned on due to the inhibition of PET. Subsequently, changes in $\mathrm{H}_{2} \mathrm{O}_{2}$ concentration during fibrosis were successfully visualized by in vitro and in vivo fluorescence imaging, which indicated the levels of oxidative stress in fibroblasts.

The peroxynitrite $\left(\mathrm{ONOO}^{-}\right)$response groups can also be reduced fluorescent dyes that show no or weak fluorescence, but exhibit intense emission after oxidation by $\mathrm{ONOO}^{-}$ (Wang et al., 2018). For example, oxazine can convert to a
NIR-fluorescent dye ( $\mathrm{SiO} 3)$ by replacing the bridging $\mathrm{O}$-atom in oxazine with a Si-atom (Wang et al., 2020). The fluorescence intensity of the reduced species (HSiO3) could be enhanced 208fold or 216-fold after treatment with hypochlorite $\left(\mathrm{ClO}^{-}\right)$or $\mathrm{ONOO}^{-}$to form SiO3. The off-on fluorescent switch performed well in fluorescence imaging of $\mathrm{HClO} / \mathrm{ONOO}^{-}$in IPF mice. In comparison with single-photon fluorescent probes, two-photon fluorescent probes usually have higher spatial resolution and imaging depth. A new two-photon NIR-fluorescent probe, rTPONOO-1, was obtained through direct condensation of acedan (two-photon fluorescent dye) and an indolium derivative (Zhan et al., 2019). Its $\mathrm{C}=\mathrm{C}$ bond could be selectively cleaved by $\mathrm{ONOO}^{-}$, causing a blue shift of the emission wavelength from 718 to $535 \mathrm{~nm}$ (Figure 2A). Twophoton imaging in frozen lung sections exhibited high resolution at penetration depth up to $110 \mu \mathrm{m}$. Moreover, the fluorescence 
A
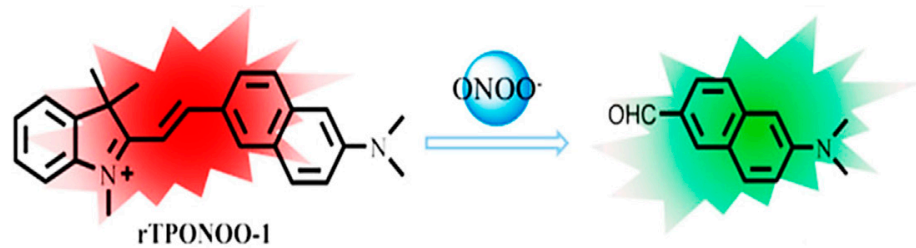

B
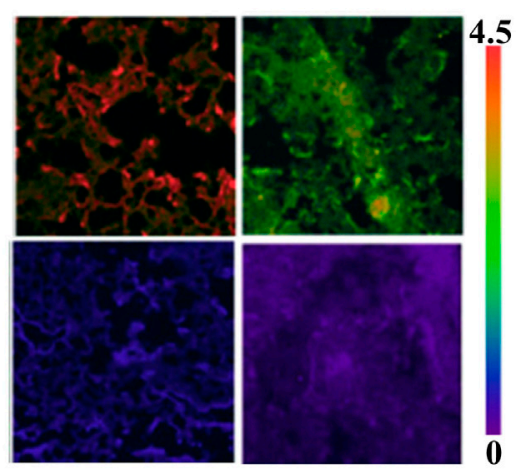

D
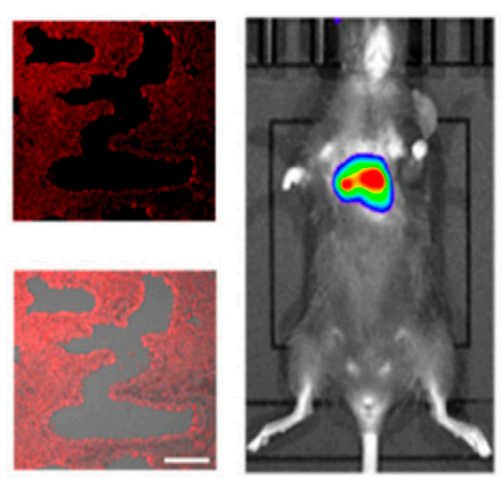

F
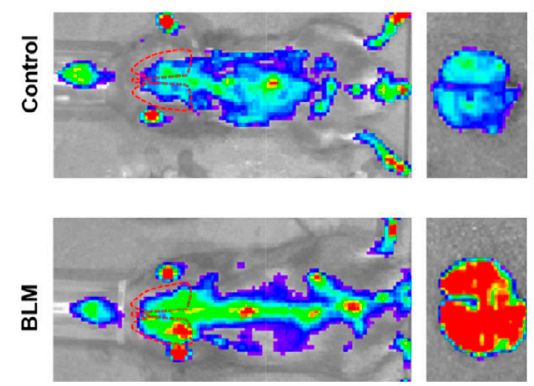

FIGURE 2 | (A) Detection mechanism of probe rTPONOO-1 to ONOO-. (B) Imaging of $\mathrm{ONOO}^{-}$levels from healthy lung slices to lung inflammation and pulmonary fibrosis. (C) Proposed Detection Mechanism of the Probe Cy-GST against GSTs. (D) The GST concentration of PF mice at 28 days of bleomycin stimulation, and the fluorescence imaging and merged imaging of fluorescence and bright-field imaging of lung slices by treatment with Cy-GST. (E) Schematic illustration of the sensitive detection of NO by the fluorescent probe PNO1. (F) Representative images of control or bleomycin mice, and the lungs isolated from indicated groups. Adapted and modified with permission from ref (He et al., 2019) (Copyright 2019 American Chemical Society), ref (Zhan et al., 2019) (Copyright 2019 American Chemical Society) and ref (Dong et al., 2020) (Copyright 2019 American Chemical Society).

ratio changes of rTPONOO-1 in bleomycin-treated mice showed a good linear relationship with the concentrations of bleomycin (Figure 2B), providing the opportunity for the early prediction of PF progression.

Compared to the emission from the NIR-I $(680-900 \mathrm{~nm})$ window, the NIR-II $(900-2000 \mathrm{~nm})$ emission has higher penetration depth and less light scattering to facilitate better imaging (Wang et al., 2019a; Zhao et al., 2019; Liang et al., 2021). For example, efficient 1,060 nm emission could be achieved using glutathione (GSH)-modified lanthanide-based nanoprobes (Zhao et al., 2019). Higher signal-to-noise ratios were achieved by the generation of cross-linked nanoprobes that accumulated at ROS-producing sites (e.g., inflammatory regions), as a result of
ROS-induced cross-linking between sulfhydryl groups in GSH. On the other hand, different from the absolute intensity mode, the absorption competition-induced emission between lanthanide-based nanoprobes and Cy7.5 fluorophores under dual-wavelength (808 and $980 \mathrm{~nm})$ excitation could show ratiometric response to $\mathrm{HClO}$ (Wang et al., 2019a). The highresolution ratiometric fluorescence imaging at $1,550 \mathrm{~nm}$ had a penetration depth of $3.5 \mathrm{~mm}$ in the scattering tissue phantom. Through the incorporation of phenyl borate group and benzothiopyrylium cyanines skeleton ( $\mathrm{Li}$ et al., 2019b), a new turn-on fluorescent probe for $\mathrm{ONOO}^{-}$(IRBTP-B) was constructed. The dioxaborolane group in the probe IRBTP-B was shown to be oxidized by $\mathrm{ONOO}^{-}$and produce a fluorophore 
with entire conjugation, which revealed a NIR-II fluorescence emission. Tissue phantom study confirmed that reliable signal was obtained at a penetration depth of up to $5 \mathrm{~mm}$.

In contrast to ROS, oxidative stress was negatively correlated with the levels of antioxidants (Pisoschi and Pop, 2015; Liu et al., 2018). GSH is a typical intracellular antioxidant and is found in relatively low concentrations in the lungs of PF patients (Wang et al., 2019b; He et al., 2020). Considering that cysteine is a raw material for GSH synthesis, cysteine levels are expected to be an indicator for the diagnosis of PF (Wang et al., 2019b). Photoacoustic and fluorescent dual-modality imaging probe CCYS consisting of a hemianthocyanine NIR-fluorescent dye and an acrylate response group was used for in situ monitoring of cysteine concentrations in PF mice. Higher cysteine concentrations were found in PF mice than in healthy mice, suggesting that low concentrations of GSH was not caused by low concentrations of cysteine. Further comparing the photoacoustic and fluorescence signals of CCYS in PF, pneumonia, and healthy mice, only $\mathrm{PF}$ mice showed a significant response, which provided a potential tool to differentiate PF from pneumonia. On the other hand, glutathione S-transferase (GST) and glutamyl transpeptidase (GGT) catalyze the nucleophilic addition of GSH to oxidative stress products and are thus potential indicators for monitoring PF progression (He et al., 2019; He et al., 2020). Two NIR fluorescent probes Cy-GST and Cy-GGT were obtained through the introducing of 3,4-dinitrobenzoic acid group (GST response unit, Figure 2C) and $\gamma$-glutamyl amide group (GGT response unit) to the cyanine (NIR-fluorescent dye). The PETinduced fluorescence quenching that occurred in Cy-GST and Cy-GGT could be selectively interrupted in the presence of GST and GGT. The specific imaging of GST and GGT concentrations in PF cells and mouse models demonstrated that higher levels of oxidative stress led to the increase of GST (Figure 2D) and GGT.

\section{IMAGING OF PULMONARY FIBROSIS-RELATED BIOMARKERS}

Cyclooxygenase-2 (COX-2) is an inducible enzyme closely related to PF (Robertson et al., 2012; Feng et al., 2019). A NIR-fluorescent probe Cy-COX, formed by covalently linking indomethacin (response group) and cyanine (NIR-fluorescent dye) with hexanediamine, has been successfully used to evaluate and image the level of COX-2 in organisms (Wang et al., 2021). The hydrophobic cavity of the COX-2 homodimer could be occupied by indomethacin, and Cy-COX adopted an unfolded conformation to suppress the PET effect with turn-on fluorescence. The imaging results of the PF mouse model showed that COX-2 played a role in alveolar cell inflammation and early PF through high expression.

Small metabolites are considered as sensitive markers for disease diagnosis (Wishart, 2016; Si and Lee, 2020). For example, the increase of dimethylarginine dimethylaminohydrolases (DDAHs) and inducible nitric oxide synthase (iNOS) could cause the up-regulation of nitric oxide (NO) expression in patients with IPF (Dong et al., 2020). The
TABLE 1 | Properties of PF-related fluorescent probes.

\begin{tabular}{|c|c|c|c|}
\hline Probe & Target & Emission wavelength & References \\
\hline Mito-Bor & $\mathrm{H}_{2} \mathrm{O}_{2}$ & $730 \mathrm{~nm}$ & Song et al. (2021) \\
\hline $\mathrm{HSiO} 3$ & $\mathrm{HClO} / \mathrm{ONOO}^{-}$ & $760 \mathrm{~nm}$ & Wang et al. (2020) \\
\hline rTPONOO-1 & $\mathrm{ONOO}^{-}$ & $718 / 535 \mathrm{~nm}$ & Zhan et al. (2019) \\
\hline Compound 1 & $\mathrm{H}_{2} \mathrm{O}_{2}$ & $667 \mathrm{~nm}$ & Liang et al. (2021) \\
\hline DCNP@GSH & $\mathrm{H}_{2} \mathrm{O}_{2}$ & $1,060 \mathrm{~nm}$ & Zhao et al. (2019) \\
\hline DCNP@Cy7.5 & $\mathrm{HClO}$ & $1,550 \mathrm{~nm}$ & Wang et al. (2019a) \\
\hline IRBTP-B & $\mathrm{ONOO}^{-}$ & $950 \mathrm{~nm}$ & Li et al. (2019b) \\
\hline CCYS & Cys & $710 \mathrm{~nm}$ & Wang et al. (2019b) \\
\hline Cy-GST & GST & $810 \mathrm{~nm}$ & He et al. (2019) \\
\hline Cy-GGT & GGT & $780 \mathrm{~nm}$ & He et al. (2020) \\
\hline Cy-COX & COX-2 & $770 \mathrm{~nm}$ & Wang et al. (2021) \\
\hline PNO1 & NO & $559 \mathrm{~nm}$ & Dong et al. (2020) \\
\hline
\end{tabular}

fluorescence of the probe PNO1 was significantly increased after treatment with NO. Its excellent sensitivity to NO enabled highcontrast imaging in diseased primary cells, tissues, living mice with bleomycin-induced fibrosis, and clinical PF tissues. Compared to the control group, the fluorescence intensity of PNO1 in the lungs of PF-affected mice was about 6 times higher, reflecting the ability of the probe PNO1 to sense local NO changes in the microenvironment (Figures 2E,F), and has a complementary effect on large-scale anti-PF drug screening. The properties of the above PF-related probes are briefly summarized in Table 1. It can be seen that most probes emit light in the NIR region, which can be used for deep tissue imaging. To further increase the penetration depth, NIR-II probes with longer emission wavelength are emerging, and promising for in vivo and in situ diagnosis of PF.

\section{IMAGING-GUIDED THERAPY}

Fluorescence imaging can be a useful technique for guiding PF therapy in addition to the detection of PF-related collagen, oxidative stress and inflammation, and biomarkers. In response to the abnormal expression of biomarkers in the process of $\mathrm{PF}$, different treatments have been proposed to help ease symptoms. The chemical structure of fluorofenidone (AKF) is similar to that of the anti-fibrotic drug pirfenidone, which can inhibit the growth of fibroblasts and the synthesis of collagen. Spermidine (Spd)-modified poly (lactic-co-glycolic acid) (PLGA) nanoparticles (NPs) were used as an AKF carrier to improve the anti-fibrosis effect (Tang et al., 2017). Coumarin-6 and 1,1'dioctadecyl-3,3,3',3'-tetramethyl indotricarbocyanine iodide (DiR) fluorophores were encapsulated in Spd-AKF-PLGA NPs for in vivo fluorescence imaging to give the distribution information of Spd-AKF-PLGA NPs during the therapy. Through the specific recognition by lung epithelial cells and accumulate in lung tissues, Spd-AKF-PLGA NPs reduced the degree of fibrosis and alveolar destruction in paraquat-induced animal models. Some ROS-sensitive fluorophore, such as nonfluorescent dihydrorhodamine-123, which can be rapidly oxidized by ROS to the strongly fluorescent rhodamine 123 , allowed determination of the ROS level using fluorescence changes (Liu et al., 2018). When antioxidants (Liu et al., 2018) 
or ROS scavengers (Zhou et al., 2018) show great potential to balance oxidative stress in PF, inhibition of ROS production in treated cells can be observed by fluorescence imaging.

Stem cells are a type of unlimited self-renewaling cells that can migrate to injured tissues and differentiate into target cells after transplantation to exert their therapeutic potential (Chen et al., 2018). In clinical trials based on stem cell therapy, bone marrow mesenchymal stem cells (BMSCs) are often used to study the treatment of PF. Transplanted BMSCs exerted anti-inflammatory and anti-fibrotic effects by down-regulating the expression of pro-inflammatory cytokines and chemokines released by macrophage activation (Huang et al., 2020). Different types of NIR fluorescent NPs (such as quantum dots, rare-earth NPs, and organic fluorescent NPs) have been used for in vivo tracking of transplanted stem cells (Chen et al., 2018). It was confirmed that BMSCs labeled with AA@ICG@PLL NPs could be effectively transplanted into $\mathrm{SiO}_{2}$-induced PF mice, and then monitored by CT/NIR fluorescence dual-modality imaging for up to 21 days (Huang et al., 2020). Additionally, BMSCs were observed to migrate to damaged lung tissue when DiR-labeled BMSCs were transplanted into a $\mathrm{SiO}_{2}$-induced PF rat model (Li et al., 2018). Optical micrographs also indicated that injection of BMSCs did reduce $\mathrm{SiO}_{2}$-induced collagen deposition and the number of nodules in lung tissue, while causing up-regulation of the expression of alveolar epithelial markers. Thus, image-guided therapy provides a useful tool for studying the mechanisms of PF and offers a bright future for the treatment of PF patients.

\section{CONCLUSION AND OUTLOOK}

On the basis of the pathological process of PF, this review covers the fluorescence imaging studies used in the last years for PF diagnosis and to assess the effect of relevant drug treatments. Currently, there are no other simple, rapid and effective diagnostic tools when HRCT or surgical lung biopsy is not possible, and the identification of biomarkers (e.g., ROS, inducible enzymes, and small metabolites) would be of great help to perform the early diagnosis of PF. The use of specific fluorescent probes to label PF-associated biomarkers allows for

\section{REFERENCES}

Bai, H., Liu, Z., Zhang, T., Du, J., Zhou, C., He, W., et al. (2020). Multifunctional Supramolecular Assemblies with Aggregation-Induced Emission (AIE) for Cell Line Identification, Cell Contamination Evaluation, and Cancer Cell Discrimination. ACS Nano 14, 7552-7563. doi:10.1021/acsnano.0c03404

Bai, Y., Zhao, J., Wang, S., Lin, T., Ye, F., and Zhao, S. (2021). Carbon Dots with Absorption Red-Shifting for Two-Photon Fluorescence Imaging of Tumor Tissue $\mathrm{pH}$ and Synergistic Phototherapy. ACS Appl. Mater. Inter. 13, 35365-35375. doi:10.1021/acsami.1c08076

Barratt, S., Creamer, A., Hayton, C., and Chaudhuri, N. (2018). Idiopathic Pulmonary Fibrosis (IPF): An Overview. Jcm 7, 201. doi:10.3390/jcm7080201

Cai, Z., Zhu, L., Wang, M., Roe, A. W., Xi, W., and Qian, J. (2020). NIR-II Fluorescence Microscopic Imaging of Cortical Vasculature in Non-human Primates. Theranostics 10, 4265-4276. doi:10.7150/thno.43533

Chen, G., Zhang, Y., Li, C., Huang, D., Wang, Q., and Wang, Q. (2018). Recent Advances in Tracking the Transplanted Stem Cells Using Near-Infrared real-time detection of fluctuating levels of these biomarkers, providing visual evidence of their abnormal expression in vivo and/or in vitro. Meanwhile, probes with NIR fluorescence emission can reduce background interference and provide high-precision imaging of deep tissues. On the other hand, multimodal imaging that combines fluorescence imaging and other imaging techniques shows great potential for generating more comprehensive information in PF detection and treatment response.

Despite some advances in fluorescence imaging for $\mathrm{PF}$ diagnosis, there are still some unresolved challenges. For example, ROS levels may be associated with other diseases and corresponding inflammation, causing the results of imaging and measuring ROS to be unspecific to PF disease. The translation of preclinical studies and in vitro tests to the clinic requires safe fluorophores and standard imaging protocols. A feasible solution is to select a suitable fluorophore among the existing Food and Drug Administration (FDA)-approved medicines as the fluorescent parent and to make reasonable modifications. Current fluorescence imaging systems also need to be further improved in order to be suitable for clinical applications. In addition, multimodal imaging may be able to accelerate the clinical translation of fluorescence imaging by using existing fluorescent probes in conjunction with other FDA-approved imaging agents.

\section{AUTHOR CONTRIBUTIONS}

All authors listed have made a substantial, direct, and intellectual contribution to the work and approved it for publication.

\section{FUNDING}

This work was supported by the National Natural Science Foundation of China (21804094 and 21804006), the Scientific Research Project of Beijing Educational Committee (KM201910028015), the Beijing Natural Science Foundation (2212013), and the Fundamental Research Funds for the Central Universities (buctrc201820).

Fluorescent Nanoprobes: Turning from the First to the Second NearInfrared Window. Adv. Healthc. Mater. 7, 1800497. doi:10.1002/ adhm.201800497

Cheresh, P., Kim, S.-J., Tulasiram, S., and Kamp, D. W. (2013). Oxidative Stress and Pulmonary Fibrosis. Biochim. Biophys. Acta (Bba) - Mol. Basis Dis. 1832, 1028-1040. doi:10.1016/j.bbadis.2012.11.021

Crisan-Dabija, R., Pavel, C. A., Popa, I. V., Tarus, A., and Burlacu, A. (2020). "A Chain Only as Strong as its Weakest Link": An Up-to-Date Literature Review on the Bidirectional Interaction of Pulmonary Fibrosis and COVID-19. J. Proteome Res. 19, 4327-4338. doi:10.1021/ acs.jproteome.0c00387

Désogère, P., Tapias, L. F., Hariri, L. P., Rotile, N. J., Rietz, T. A., Probst, C. K., et al. (2017). Type I Collagen-Targeted PET Probe for Pulmonary Fibrosis Detection and Staging in Preclinical Models. Sci. Transl. Med. 9, eaaf4696. doi:10.1126/ scitranslmed.aaf4696

Dong, Y., Li, X.-R., Chen, Q., Guo, R.-Y., Tang, B.-X., Kan, W.-J., et al. (2020). Microenvironment-Responsive Small-Molecule Probe for Pulmonary Fibrosis Detection. Anal. Chem. 92, 699-706. doi:10.1021/acs.analchem.9b02264 
Fatahi, Z., Esfandiari, N., and Ranjbar, Z. (2020). A New Anti-counterfeiting Feature Relying on Invisible Non-toxic Fluorescent Carbon Dots. J. Anal. Test. 4, 307-315. doi:10.1007/s41664-020-00149-6

Feng, F., Wang, Z., Li, R., Wu, Q., Gu, C., Xu, Y., et al. (2019). Citrus Alkaline Extracts Prevent Fibroblast Senescence to Ameliorate Pulmonary Fibrosis via Activation of COX-2. Biomed. Pharmacother. 112, 108669. doi:10.1016/ j.biopha.2019.108669

Fligiel, S. E. G., Varani, J., Datta, S. C., Kang, S., Fisher, G. J., and Voorhees, J. J. (2003). Collagen Degradation in Aged/Photodamaged Skin In Vivo and after Exposure to Matrix Metalloproteinase-1 In Vitro. J. Invest. Dermatol. 120, 842-848. doi:10.1046/j.1523-1747.2003.12148.x

George, P. M., Wells, A. U., and Jenkins, R. G. (2020). Pulmonary Fibrosis and COVID-19: The Potential Role for Antifibrotic Therapy. Lancet Respir. Med. 8, 807-815. doi:10.1016/S2213-2600(20)30225-3

Guan, W., Yang, T., and Lu, C. (2020). Measurement of Solubilization Location in Micelles Using Anchored Aggregation-Induced Emission Donors. Angew. Chem. Int. Ed. 59, 12800-12805. doi:10.1002/anie.202005085

Guo, L., Tian, M., Zhang, Z., Lu, Q., Liu, Z., Niu, G., et al. (2021). Simultaneous Two-Color Visualization of Lipid Droplets and Endoplasmic Reticulum and Their Interplay by Single Fluorescent Probes in Lambda Mode. J. Am. Chem. Soc. 143, 3169-3179. doi:10.1021/jacs.0c12323

Han, H.-H. H. T., Jr, Tian, H., Zang, Y., Sedgwick, A. C., Li, J., Sessler, J. L., et al. (2021). Small-Molecule Fluorescence-Based Probes for Interrogating Major Organ Diseases. Chem. Soc. Rev. 50, 9391-9429. doi:10.1039/d0cs01183e

Hayward, K. L., Kouthouridis, S., and Zhang, B. (2021). Organ-on-a-Chip Systems for Modeling Pathological Tissue Morphogenesis Associated with Fibrosis and Cancer. ACS Biomater. Sci. Eng. 7, 2900-2925. doi:10.1021/ acsbiomaterials.0c01089

He, N., Bai, S., Huang, Y., Xing, Y., Chen, L., Yu, F., et al. (2019). Evaluation of Glutathione S-Transferase Inhibition Effects on Idiopathic Pulmonary Fibrosis Therapy with a Near-Infrared Fluorescent Probe in Cell and Mice Models. Anal. Chem. 91, 5424-5432. doi:10.1021/acs.analchem.9b00713

He, N., Wang, Y., Huang, Y., Wang, X., Chen, L., and Lv, C. (2020). A NearInfrared Fluorescent Probe for Evaluating Glutamyl Transpeptidase Fluctuation in Idiopathic Pulmonary Fibrosis Cell and Mice Models. Sensors Actuators B: Chem. 322, 128565. doi:10.1016/j.snb.2020.128565

He, W., Zheng, Z., Bai, H., Xiong, L.-H., Wang, L., Li, Y., et al. (2021). A Biocompatible Dual-AIEgen System without Spectral Overlap for Quantitation of Microbial Viability and Monitoring of Biofilm Formation. Mater. Horiz. 8, 1816-1824. doi:10.1039/d1mh00149c

Hong, G., Zou, Y., Antaris, A. L., Diao, S., Wu, D., Cheng, K., et al. (2014). Ultrafast Fluorescence Imaging in Vivo with Conjugated Polymer Fluorophores in the Second Near-Infrared Window. Nat. Commun. 5, 4206. doi:10.1038/ ncomms5206

Huang, J., Huang, J., Ning, X., Luo, W., Chen, M., Wang, Z., et al. (2020). CT/NIRF Dual-Modal Imaging Tracking and Therapeutic Efficacy of Transplanted Mesenchymal Stem Cells Labeled with Au Nanoparticles in Silica-Induced Pulmonary Fibrosis. J. Mater. Chem. B 8, 1713-1727. doi:10.1039/c9tb02652e

Hwang, J., Huang, Y., Burwell, T. J., Peterson, N. C., Connor, J., Weiss, S. J., et al. (2017). In Situ Imaging of Tissue Remodeling with Collagen Hybridizing Peptides. ACS Nano 11, 9825-9835. doi:10.1021/acsnano.7b03150

Hwang, J., San, B. H., Turner, N. J., White, L. J., Faulk, D. M., Badylak, S. F., et al. (2017). Molecular Assessment of Collagen Denaturation in Decellularized Tissues Using a Collagen Hybridizing Peptide. Acta Biomater. 53, 268-278. doi:10.1016/j.actbio.2017.01.079

Juillerat-Jeanneret, L., Aubert, J.-D., Mikulic, J., and Golshayan, D. (2018). Fibrogenic Disorders in Human Diseases: From Inflammation to Organ Dysfunction. J. Med. Chem. 61, 9811-9840. doi:10.1021/acs.jmedchem.8b00294

Ley, S., and Ley-Zaporozhan, J. (2020). Novelties in Imaging in Pulmonary Fibrosis and Nodules. A Narrative Review. Pulmonology 26, 39-44. doi:10.1016/ j.pulmoe.2019.09.009

Li, C., Chen, G., Zhang, Y., Wu, F., and Wang, Q. (2020). Advanced Fluorescence Imaging Technology in the Near-Infrared-II Window for Biomedical Applications. J. Am. Chem. Soc. 142, 14789-14804. doi:10.1021/jacs.0c07022

Li, D., Wang, S., Lei, Z., Sun, C., El-Toni, A. M., Alhoshan, M. S., et al. (2019). Peroxynitrite Activatable NIR-II Fluorescent Molecular Probe for DrugInduced Hepatotoxicity Monitoring. Anal. Chem. 91, 4771-4779. doi:10.1021/acs.analchem.9b00317
Li, X., An, G., Wang, Y., Liang, D., Zhu, Z., and Tian, L. (2018). Targeted Migration of Bone Marrow Mesenchymal Stem Cells Inhibits Silica-Induced Pulmonary Fibrosis in Rats. Stem Cel Res. Ther. 9, 1-13. doi:10.1186/s13287-018-1083-y

Li, X., Shi, L., Li, L., Dong, C., Li, C.-Z., and Shuang, S. (2019). Recent Advances in Carbon Nanodots: Properties and Applications in Cancer Diagnosis and Treatment. J. Anal. Test. 3, 37-49. doi:10.1007/s41664-019-00089-w

Liang, T., Zhang, D., Hu, W., Tian, C., Zeng, L., Wu, T., et al. (2021). A Dual Lockand-Key Two Photon Fluorescence Probe in Response to Hydrogen Peroxide and Viscosity: Application in cellular Imaging and Inflammation Therapy. Talanta 235, 122719. doi:10.1016/j.talanta.2021.122719

Liu, M., Xu, H., Zhang, L., Zhang, C., Yang, L., Ma, E., et al. (2018). Salvianolic Acid B Inhibits Myofibroblast Transdifferentiation in Experimental Pulmonary Fibrosis via the Up-Regulation of Nrf2. Biochem. Biophysical Res. Commun. 495, 325-331. doi:10.1016/j.bbrc.2017.11.014

Liu, Y.-M., Nepali, K., and Liou, J.-P. (2017). Idiopathic Pulmonary Fibrosis: Current Status, Recent Progress, and Emerging Targets. J. Med. Chem. 60, 527-553. doi:10.1021/acs.jmedchem.6b00935

Liu, Z., Zou, H., Zhao, Z., Zhang, P., Shan, G.-G., Kwok, R. T. K., et al. (2019). Tuning Organelle Specificity and Photodynamic Therapy Efficiency by Molecular Function Design. ACS Nano 13, 11283-11293. doi:10.1021/ acsnano.9b04430

Lonzetti, L., Zanon, M., Pacini, G. S., Altmayer, S., Martins de Oliveira, D., Rubin, A. S., et al. (2019). Magnetic Resonance Imaging of Interstitial Lung Diseases: A State-of-the-Art Review. Respir. Med. 155, 79-85. doi:10.1016/j.rmed.2019.07.006

Ma, Z., Zhang, M., Yue, J., Alcazar, C., Zhong, Y., Doyle, T. C., et al. (2018). NearInfrared IIb Fluorescence Imaging of Vascular Regeneration with Dynamic Tissue Perfusion Measurement and High Spatial Resolution. Adv. Funct. Mater. 28, 1803417. doi:10.1002/adfm.201803417

Mammarappallil, J. G., Rankine, L., Wild, J. M., Driehuys, B., Wild, J., and Driehuys, B. (2019). New Developments in Imaging Idiopathic Pulmonary Fibrosis with Hyperpolarized Xenon Magnetic Resonance Imaging. J. Thorac. Imaging 34, 136-150. doi:10.1097/RTI.0000000000000392

Matteini, P., Cicchi, R., Ratto, F., Kapsokalyvas, D., Rossi, F., de Angelis, M., et al. (2012). Thermal Transitions of Fibrillar Collagen Unveiled by SecondHarmonic Generation Microscopy of Corneal Stroma. Biophysical J. 103, 1179-1187. doi:10.1016/j.bpj.2012.07.055

Mento, F., Soldati, G., Prediletto, R., Demi, M., and Demi, L. (2020). Quantitative Lung Ultrasound Spectroscopy Applied to the Diagnosis of Pulmonary Fibrosis: The First Clinical Study. IEEE Trans. Ultrason. Ferroelect., Freq. Contr. 67, 2265-2273. doi:10.1109/TUFFC.2020.3012289

Nakajima, Y., Asano, K., Mukai, K., Urai, T., Okuwa, M., Sugama, J., et al. (2018). Near-Infrared Fluorescence Imaging Directly Visualizes Lymphatic Drainage Pathways and Connections between Superficial and Deep Lymphatic Systems in the Mouse Hindlimb. Sci. Rep. 8, 1-9. doi:10.1038/s41598-018-25383-y

Ni, K., Liu, M., Zheng, J., Wen, L., Chen, Q., Xiang, Z., et al. (2018). PD-1/PD-L1 Pathway Mediates the Alleviation of Pulmonary Fibrosis by Human Mesenchymal Stem Cells in Humanized Mice. Am. J. Respir. Cel Mol Biol 58, 684-695. doi:10.1165/rcmb.2017-0326OC

Nielsen, L. F., Moe, D., Kirkeby, S., and Garbarsch, C. (1998). Sirius Red and Acid Fuchsin Staining Mechanisms. Biotech. Histochem. 73, 71-77. doi:10.3109/ 10520299809140509

Niu, G., Zhang, R., Shi, X., Park, H., Xie, S., Kwok, R. T. K., et al. (2020). AIE Luminogens as Fluorescent Bioprobes. Trac Trends Anal. Chem. 123, 115769. doi:10.1016/j.trac.2019.115769

Perez, J. R., Ybarra, N., Chagnon, F., Serban, M., Pare, G., Lesur, O., et al. (2017). Image-Guided Fluorescence Endomicroscopy: From Macro- to Micro-imaging of Radiation-Induced Pulmonary Fibrosis. Sci. Rep. 7, 1-11. doi:10.1038/ s41598-017-18070-x

Pisoschi, A. M., and Pop, A. (2015). The Role of Antioxidants in the Chemistry of Oxidative Stress: A Review. Eur. J. Med. Chem. 97, 55-74. doi:10.1016/ j.ejmech.2015.04.040

Richeldi, L., Collard, H. R., and Jones, M. G. (2017). Idiopathic Pulmonary Fibrosis. The Lancet 389, 1941-1952. doi:10.1016/S0140-6736(17)30866-8

Robertson, J. A., Sauer, D., Gold, J. A., and Nonas, S. A. (2012). The Role of Cyclooxygenase-2 in Mechanical Ventilation-Induced Lung Injury. Am. J. Respir. Cel Mol Biol 47, 387-394. doi:10.1165/rcmb.2011-0005OC

Ruscitti, F., Ravanetti, F., Donofrio, G., Ridwan, Y., van Heijningen, P., Essers, J., et al. (2018). A Multimodal Imaging Approach Based on Micro-CT and 
Fluorescence Molecular Tomography for Longitudinal Assessment of Bleomycin-Induced Lung Fibrosis in Mice. JoVE 134, e56443. doi:10.3791/ 56443

Schieber, M., and Chandel, N. S. (2014). ROS Function in Redox Signaling and Oxidative Stress. Curr. Biol. 24, R453-R462. doi:10.1016/j.cub.2014.03.034

$\mathrm{Si}, \mathrm{Y}$., and Lee, H. J. (2020). Carbon Nanomaterials and Metallic NanoparticlesIncorporated Electrochemical Sensors for Small Metabolites: Detection Methodologies and Applications. Curr. Opin. Electrochemistry 22, 234-243. doi:10.1016/j.coelec.2020.08.007

Snijder, J., Peraza, J., Padilla, M., Capaccione, K., and Salvatore, M. M. (2019). Pulmonary Fibrosis: A Disease of Alveolar Collapse and Collagen Deposition. Expert Rev. Respir. Med. 13, 615-619. doi:10.1080/17476348.2019.1623028

Song, X., Bai, S., He, N., Wang, R., Xing, Y., Lv, C., et al. (2021). Real-Time Evaluation of Hydrogen Peroxide Injuries in Pulmonary Fibrosis Mice Models with a Mitochondria-Targeted Near-Infrared Fluorescent Probe. ACS Sens. 6, 1228-1239. doi:10.1021/acssensors.0c02519

Sorushanova, A., Delgado, L. M., Wu, Z., Shologu, N., Kshirsagar, A., Raghunath, R., et al. (2019). The Collagen Suprafamily: From Biosynthesis to Advanced Biomaterial Development. Adv. Mater. 31, 1801651. doi:10.1002/ adma.201801651

Tang, J., Li, J., Li, G., Zhang, H., Wang, L., Li, D., et al. (2017). SpermidineMediated Poly(lactic-co-Glycolic Acid) Nanoparticles Containing Fluorofenidone for the Treatment of Idiopathic Pulmonary Fibrosis. Ijn Vol. 12, 6687-6704. doi:10.2147/IJN.S140569

Wan, H., Yue, J., Zhu, S., Uno, T., Zhang, X., Yang, Q., et al. (2018). A Bright Organic NIR-II Nanofluorophore for Three-Dimensional Imaging into Biological Tissues. Nat. Commun. 9, 1171. doi:10.1038/s41467-018-03505-4

Wang, H., Zhang, Y., Yang, Y., He, Z., Wu, C., Zhang, W., et al. (2019). In Situ Photoacoustic Imaging of Cysteine to Reveal the Mechanism of Limited GSH Synthesis in Pulmonary Fibrosis. Chem. Commun. 55, 9685-9688. doi:10.1039/ c9cc03814k

Wang, L., Liu, J., Zhao, S., Zhang, H., Sun, Y., Wei, A., et al. (2020). Fluorescence Imaging of Hypochlorous Acid and Peroxynitrite In Vitro and In vivo with Emission Wavelength Beyond $750 \mathrm{~nm}$. Chem. Commun. 56, 7718-7721. doi:10.1039/d0cc02322a

Wang, S., Chen, L., Jangili, P., Sharma, A., Li, W., Hou, J.-T., et al. (2018). Design and Applications of Fluorescent Detectors for Peroxynitrite. Coord. Chem. Rev. 374, 36-54. doi:10.1016/j.ccr.2018.06.013

Wang, S., Liu, L., Fan, Y., El-Toni, A. M., Alhoshan, M. S., Li, D., et al. (2019). In Vivo High-resolution Ratiometric Fluorescence Imaging of Inflammation Using NIR-II Nanoprobes with $1550 \mathrm{~nm}$ Emission. Nano Lett. 19, 2418-2427. doi:10.1021/acs.nanolett.8b05148

Wang, Y., Wei, Y., He, N., Zhang, L., You, J., Chen, L., et al. (2021). Evaluation of Cyclooxygenase-2 Fluctuation via a Near-Infrared Fluorescent Probe in Idiopathic Pulmonary Fibrosis Cell and Mice Models. J. Mater. Chem. B 9, 6226-6233. doi:10.1039/d1tb01307f

Wei, Y., Liu, Y., He, Y., and Wang, Y. (2021). Mitochondria and LysosomeTargetable Fluorescent Probes for Hydrogen Peroxide. J. Mater. Chem. B 9, 908-920. doi:10.1039/d0tb02440f
Wishart, D. S. (2016). Emerging Applications of Metabolomics in Drug Discovery and Precision Medicine. Nat. Rev. Drug Discov. 15, 473-484. doi:10.1038/ $\operatorname{nrd} .2016 .32$

Wu, X., Kim, G. H., Salisbury, M. L., Barber, D., Bartholmai, B. J., Brown, K. K., et al. (2019). Computed Tomographic Biomarkers in Idiopathic Pulmonary Fibrosis. The Future of Quantitative Analysis. Am. J. Respir. Crit. Care Med. 199, 12-21. doi:10.1164/rccm.201803-0444PP

Ximendes, E., Benayas, A., Jaque, D., and Marin, R. (2021). Quo Vadis, Nanoparticle-Enabled In Vivo Fluorescence Imaging. ACS Nano 15, 1917-1941. doi:10.1021/acsnano.0c08349

Xu, W., Wang, D., and Tang, B. Z. (2021). NIR-II AIEgens: A Win-Win Integration towards Bioapplications. Angew. Chem. Int. Ed. 60, 7476-7487. doi:10.1002/ anie. 202005899

Yombo, D. J. K., Odayar, V., Gupta, N., Jegga, A. G., and Madala, S. K. (2021). The Protective Effects of IL-31RA Deficiency during Bleomycin-Induced Pulmonary Fibrosis. Front. Immunol. 12, 682. doi:10.3389/fimmu.2021.645717

Zhan, Z., Liu, R., Chai, L., Dai, Y., and Lv, Y. (2019). Visualization of Lung Inflammation to Pulmonary Fibrosis via Peroxynitrite Fluctuation. Anal. Chem. 91, 11461-11466. doi:10.1021/acs.analchem.9b02971

Zhao, M., Wang, R., Li, B., Fan, Y., Wu, Y., Zhu, X., et al. (2019). Precise In Vivo Inflammation Imaging Using In Situ Responsive Cross-linking of GlutathioneModified Ultra-small NIR-II Lanthanide Nanoparticles. Angew. Chem. Int. Ed. 58, 2050-2054. doi:10.1002/anie.201812878

Zheng, Z., Li, D., Liu, Z., Peng, H. Q., Sung, H. H. Y., Kwok, R. T. K., et al. (2019). Aggregation-Induced Nonlinear Optical Effects of AIEgen Nanocrystals for Ultradeep In Vivo Bioimaging. Adv. Mater. 31, 1904799. doi:10.1002/ adma.201904799

Zhou, Y., Zhen, M., Ma, H., Li, J., Shu, C., and Wang, C. (2018). Inhalable Gadofullerenol/[70] Fullerenol as High-Efficiency ROS Scavengers for Pulmonary Fibrosis Therapy. Nanomedicine: Nanotechnology, Biol. Med. 14, 1361-1369. doi:10.1016/j.nano.2018.03.008

Conflict of Interest: The authors declare that the research was conducted in the absence of any commercial or financial relationships that could be construed as a potential conflict of interest.

Publisher's Note: All claims expressed in this article are solely those of the authors and do not necessarily represent those of their affiliated organizations, or those of the publisher, the editors and the reviewers. Any product that may be evaluated in this article, or claim that may be made by its manufacturer, is not guaranteed or endorsed by the publisher.

Copyright $\odot 2021 \mathrm{Liu}$, Tang, Zhu, Ma, Zhou and Guan. This is an open-access article distributed under the terms of the Creative Commons Attribution License (CC $B Y)$. The use, distribution or reproduction in other forums is permitted, provided the original author(s) and the copyright owner(s) are credited and that the original publication in this journal is cited, in accordance with accepted academic practice. No use, distribution or reproduction is permitted which does not comply with these terms. 